\title{
TAUX D'ÉCLOSION ET POSITION DE L'CEUF DANS LA SÉRIE CHEZ LA POULE
}

\author{
Ph. MERAT et L. LACASSAGNE \\ Station de Recherches avicoles, \\ Centre national de Recherches zootechniques, Jouy-en-Josas.
}

\begin{abstract}
SOMMAIRE
Au printemps 1959, les taux d'éclosion de 5500 œufs ont été comparés en fonction de leur place dans la série de ponte. Les conclusions sont les suivantes :

I $^{0}$ La place d'un ouf dans la série est sans relation avec son éclosivité.

$2^{0}$ Les œufs appartenant à des séries d'une unité éclosent moins bien, et ceux provenant de séries de plus de 7 œufs éclosent mieux, que les autres.

$3^{0}$ Les cufs pondus immédiatement avant ou après un arrêt de ponted'au moins a jours éclosent moins bien que la moyenne générale.
\end{abstract}

Il semble prouvé que le poids des réserves vitellines des ovules d'une même série décroît du début vers la fin de la série (BASTIAN et ZARRow, I955; LACASSAGNE, I960). Cette disparité des réserves par ovule fait penser à l'existence d'un potentiel d'éclosion différent entre les ovules d'une même série.

Plusieurs études ont déjà été effectuées pour vérifier cette hypothèse. Hưri et PILKEY , I930, trouvent que les œufs pondus l'après-midi éclosent moins bien que ceux pondus dans la matinée. Ce qui revient à dire, étant donné le mode de ponte de la poule, que les derniers oufs de la série éclosent moins bien que les premiers. Mais FUNK, I934, Mc NALLY et BYERLY I936, arrivent à des conclusions opposées. Enfin Hays 1936, I937, FUNK I939, ne trouvent aucun rapport entre l'heure de ponte et le taux d'éclosion.

Ces résultats contradictoires nous ont incités à reprendre cette étude. Nous avons donc, au cours du printemps I959, relevé le taux d'éclosion des œufs pondus par un troupeau, en fonction de leur place dans la série. Les poules contrôlées se trouvant en parquet pedigree, nous n'avons pas analysé l'influence possible de la fréquence des accouplements sur l'éclosivité. Bien que 2 p. Ioo des œufs aient échappé au contrôle au nid-trappe, les chiffres du tableau I peuvent dans leur ensemble être considérés comme exacts. 
TABLEAU I

Éclosion suivant la position dans la série - Printemps 1959

\begin{tabular}{|c|c|c|c|c|c|}
\hline \multirow{2}{*}{$\underset{\text { série }}{\text { Rang dans la }}$} & \multicolumn{5}{|c|}{ Pourcentages d'éclosion (poussins nés/œufs incubés) } \\
\hline & séries de 2 & séries de 3 & séries de 4 & séries de 5 & séries de plus de 5 \\
\hline $\begin{array}{l}1 \\
2 \\
3 \\
4 \\
5\end{array}$ & $\begin{array}{l}75,2 \\
79,1\end{array}$ & $\begin{array}{l}73,2 \\
74,8 \\
76,4\end{array}$ & $\begin{array}{l}78,5 \\
75,9 \\
76,6 \\
76,2\end{array}$ & $\begin{array}{l}74,8 \\
73,1 \\
75.5 \\
76,9 \\
77,4\end{array}$ & $\begin{array}{c}1^{\text {er }}: 78,0 \\
\text { intermédiaires : } \\
77,2 \\
\text { dernier : } \\
73,0\end{array}$ \\
\hline
\end{tabular}

Aucune hétérogénéité n'apparaît à l'intérieur de chaque série.

L'examen des résultats obtenus montre un comportement identique des œufs au cours de l'incubation quelle que soit leur place dans la série. Les réserves d'un ovule ne semblent donc pas avoir d'influence sur son pouvoir d'éclosion. Il est à noter, à ce propos, que NEHER et FRAPS I946 avaient obtenu un développement embryonnaire apparemment normal à partir d'œufs provenant d'ovulations prématurées, qui ne possédaient pas, selon toute vraisemblance, des réserves vitellines normales.

Le classement des œutfs, non plus à l'intérieur de la série mais par séries, tableau 2, montre l'existence d'une différence significative du taux d'éclosion suivant la longueur de cette série : les œufs pondus isolément éclosent moins bien que ceux des séries de $2,3,4,5,6$ et 7 œufs. Dans les séries de plus de 7 œufs le taux d'éclosion est nettement meilleur.

TABLEAU 2

Éclosion par séries - Printemps 1959.

\begin{tabular}{|c|c|c|c|c|c|c|c|c|}
\hline $\begin{array}{c}\text { Taille de la } \\
\text { série }\end{array}$ & 1 & 2 & 3 & 4 & 5 & 6 & 7 & Plus de 7 \\
\hline OEufs clairs ... & 20 & 86 & 84 & 55 & 32 & 14 & 24 & \\
\hline Eufs morts... & 101 & 183 & 220 & 155 & 96 & 95 & 42 & 119 \\
\hline CEufs éclos ... & 241 & 849 & 911 & 692 & 395 & 267 & 231 & 588 \\
\hline Total......... & 362 & 1118 & 1215 & 902 & 523 & 376 & 297 & 707 \\
\hline $\begin{array}{l}\text { Poussins nés/ } \\
\text { œufs incubés. }\end{array}$ & & & & & & & & \\
\hline p. $100 \ldots \ldots$ & 65,4 & 76,0 & 74,7 & 76,7 & 75,5 & 71,0 & 77,7 & 83,2 \\
\hline
\end{tabular}

Hétérogénéité entre séries de 1 , de 2 à 7 inclus, et $>7: \chi^{2}$ avec 2 D. L. $=38,00 \mathrm{P}<0,001$

FUNK en 1939 et LAMMOREuX en I940 avaient trouvé un taux d'éclosion inférieur pour les œufs des séries de I et 2 œufs. Nous ne retrouvons donc que partiellement leurs résultats puisque dans notre troupeau nous n'avons pu individualiser le comportement des séries de 2 œufs. Mais dans leur ensemble nos conclusions reviennent à dire qu'il existe une corrélation positive entre le pouvoir d'éclosion et l'intensité de ponte au même moment. Ceci est cn accord avec les travaux de nombreux auteurs : JuLL, I930; BYERLY, TITUS et EIIIS I933; FUnK I934; BERNIER'I947 ; BERNIER, TAYIOR et GuNNS I95I. 
Nous avons examiné d'autre part le pourcentage d'éclosion des œufs pondus immédiatement avant ou juste après un arrêt de ponte de 2 jours et plus (appelé conventionnellement " pause »). Les résultats sont contenus dans le tableau 3 .

TABLEAU 3

Éclosion des cufs voisins d'une pause - Printemps 1959.

\begin{tabular}{|c|c|c|c|c|c|c|c|c|}
\hline \multirow{2}{*}{$\begin{array}{l}\text { Durée de l'arrêt } \\
\quad \text { (en jours) }\end{array}$} & \multicolumn{4}{|c|}{ Premier œuf après une "pause " } & \multicolumn{4}{|c|}{ Dernier œuf avant une "pause" } \\
\hline & 2 & 3 & Plus de 3 & Total & 2 & 3 & plus de 3 & Total \\
\hline $\begin{array}{l}\text { Eufs incubés } \\
\text { éclos p. } 100 . .\end{array}$ & $\begin{array}{l}204 \\
70,0\end{array}$ & $\begin{array}{l}54 \\
66,7\end{array}$ & $\begin{array}{l}55 \\
50,9\end{array}$ & $\begin{array}{l}313 \\
66,1\end{array}$ & $\begin{array}{l}198 \\
70,2\end{array}$ & $\begin{array}{l}51 \\
74,5\end{array}$ & $\begin{array}{l}55 \\
49,1\end{array}$ & $\begin{array}{l}304 \\
67,1\end{array}$ \\
\hline
\end{tabular}

Le pourcentage d'éclosion sur l'ensemble du troupeau est de 75,9 p. Ioo (sur 5500 œufs incubés), ce qui rend évident l'effet dépressif sur l'éclosion du voisinage d'une " pause " (pourcentage d'éclosion égal à 66,6 p. Ioo au total) ; et ce, d'autant plus apparemment que la "pause » est plus longue $\left(\chi^{2}\right.$ d'hétérogénéité avec $2 \mathrm{D}$. L. = respectivement 7,2 et I0,2 pour chacune des 2 moitiés du tableau $n^{0} 3 ; \mathrm{P}<0,05$ et $\mathrm{P}<\mathrm{o}$,oI respectivement.)

Cet effet s'observe encore sur un autre troupeau mis en reproduction à l'automne I959, où le pourcentage global d'éclosion est de 66,2 p. Ioo (sur 3872 œufs incubés) et l'éclosion des cufs voisins d'une " pause " est de 55, I p. Ioo, Dans ce troupeau, d'ailleurs, les poules ayant eu au moins une " pause " avaient de toutes façons un pourcentage d'éclosion global de 57,7 p. Ioo, inférieur à la moyenne du troupeau.

Ces résultats sont assez voisins de ceux de HAYs I938, qui trouve que le pourcentage d'éclosion diminue quand l'intervalle entre séries augmente.

Reçu en février 1960

\section{SUMMARY}

\section{RELATIONSHIP BETWEEN TIIE HATCHABILITY OF EGGS AND THEIR POSITION IN THE CLUTCH}

In spring 1959, the hatchability of 5500 eggs was compared to their position in the clutch. The conclusions are the following :

r. The position in the clutch bears no relation to hatchability.

2. One-egg clutches hatch poorer, and more than 7 egg-clutches hatch better, than the others.

3. Eggs laid just before or after a " pause" ( $\geqslant 2$ days) hatch poorer than the general mean.

\section{RÉFÉRENCES BIBLIOGRAPHIQUES}

Bastian J. W., Zarrow M. X., I955. A new hypothesis for the asynchronous ovulatory cycle of the domestic hen (Gallus domesticus). Ponll. Sci., 34, $77^{6-788 .}$

BERNIER P. E., T947. The relative effects of inbreeding and outbreeding on reproduction in the domestic fowl. Thesis Univ of Calif.

Bernier P. E., TAYLor L. W., Gunns C. A., I95I. The relative effects of inbreeding and outbreeding on reproduction in the domestic fowl. Hilgardia, 20, 529-628.

Byerly T. C., Titus H. W., Ellis N. R., I933. Production and hatchability of eggs as affected by different kinds and quantities of proteins in the diet of laying hens. J. Agr. Res., 46, I-22.

Funk E. M., r934. Relation of time of laying to hatchability. Poult. Sci., 13, 184-187. 
FUNK E. M., 1939. The relation of size of clutch and position of the egg in the clutch to hatching results. Poult. Sci., 18, 350-353.

HAYs F. A., I936. Time interval between eggs of Rhode Island Red pullets. I. Agr. Res., 52,633-638.

HAYs F. A, I937. Hatchability as related to seasons and hour of laying. Poull. Sci., 16, $85-80$.

HAYS F. A., I 938 . Time interval between clutches in Rhode Island Red pullets. J. Agr. Res., 57, 575-58r.

HutT F. B., PILKex A. M., I 930. Studies in embryonic mortality in the fowl. IV. Comparative mortality rates in eggs laid at different periods of the day and their bearing on theories of the origin of monsters. Poull. Sci., 9, I94-203.

JULL M. A., I930. Studies in hatchability. VI. Hatchability in relation to current egg production. Poull. Sci., 10, 327-331.

LACASSAG:E L., ig6o. Étude comparée des réserves vitellines et de la durée de la phase de grand accroissesement de l'ovocyte chez la poule domestique. Infuence de l'âge de l'animal et de la longueur de la séric. Ann. Zooted ., $1,8_{5}-96$.

LAMOREUX $1:$ F., I940. The influence of intensity of egg production upon infertility in the domestic fowl. J. Agr. Res., 6i, I9I-206.

Mc Nalli I., Bierly T. C., I936. Variation in the development of embryos of hen's eggs. Poult. Sici., 15, $280-28_{3}$.

NeHER B. H., FrAPs R. M., I946. Fertility and hatchability of the prematuraly ovulated hen's egg. J. Exp. Zoot., 101, 83-89. 